\title{
Maternal and Neonatal Tetanus Elimination: Where are We Now?
}

\author{
Shashi Kant Dhir' \\ Pooja Dewan ${ }^{2}$ \\ Piyush Gupta (iD) ${ }^{2}$
}

'Department of Pediatrics, Guru Gobind Singh Medical College, Punjab, India;

${ }^{2}$ Department of Pediatrics, University

College of Medical Sciences, Delhi, India

\section{Video abstract}

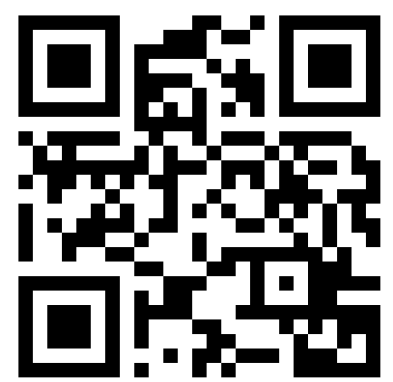

Point your SmartPhone at the code above. If you have a $Q R$ code reader the video abstract will appear. Or use: https://youtu.be/bLdnRBOKLIU

Correspondence: Piyush Gupta Department of Pediatrics, University College of Medical Sciences, Delhi, India Tel +919811597172

Fax +9|-I I-22590495

Email piyush.gupta@gmail.com

\begin{abstract}
The maternal and neonatal tetanus elimination (MNTE) program was envisaged by the World Health Organization to overcome the mortality and morbidity caused by maternal and neonatal tetanus (MNT). Although preventable by simple cost-effective practices like universal immunization, clean delivery practices, and healthy umbilical cord care, as of date MNT is still prevalent in 12 developing countries of Asia and Africa. Definitive approaches need to be microplanned by these countries to successfully accomplish the three stages of MNTE, ie, achieving, validating, and sustaining. Once a country achieves MNTE, this status is required to be validated and sustained according to the high-risk and low-risk categorization of the districts. The three-pronged strategies for achieving and sustaining MNTE include (a) rigorous immunization of women of reproductive age with tetanus toxoidcontaining vaccines, (b) strengthening of clean delivery services for pregnant women, and (c) effective surveillance for MNT. Although the deadlines for achieving MNTE globally have been missed many times, yet there has been a significant progress to date as evident by $80 \%$ reduction in countries requiring validation for MNTE (59 countries in 1999 to 12 countries in 2020). Huge strides have been made in the overall coverage of two doses of tetanus toxoid ( $13.79 \%$ to $65.27 \%)$, neonates being protected at birth (12\% to $88 \%)$, global coverage of third-dose DPT (more than doubled), and reduction of $88 \%$ estimated deaths due to NT in the last four decades. Identification of the most vulnerable populations, systematic planning at all levels of health care, involvement of local community support, tackling the implementation gap, strong political will, good financial support, and continued robust surveillance will go a long way in achieving MNTE.
\end{abstract}

Keywords: tetanus toxoid containing vaccines, clean delivery, maternal tetanus, neonatal tetanus, strategy, tetanus immunization, MNTE validation

\section{Introduction}

Tetanus is caused by a Gram-positive, rod-shaped, spore-bearing obligate anaerobe, Clostridium tetani. Spores of bacterium enter via breached skin and germinate in the presence of anaerobic environment in unprotected individuals. ${ }^{1}$ Highly potent neurotoxin is produced locally and then transported to the central nervous system via the retrograde axonal transport system. The toxin acts on the peripheral motor end plates, spinal cord, brain, and on the autonomic nervous system and disrupts the inhibitory interneurons causing unopposed excitation by $\alpha$ motor neurons. ${ }^{2}$

Maternal tetanus (MT) may occur after miscarriage, unsafe abortion, or unhygienic delivery. Neonatal tetanus (NT) may occur due to unhealthy umbilical cord care practices. ${ }^{3}$ The incubation period of tetanus varies between a few days and several weeks. NT has shorter incubation as compared to MT, and the majority of 
neonates develop symptoms within 3 to 14 days of insult, mostly 6-8 days. ${ }^{4}$ The diagnosis of tetanus is clinical and is based on physical examination signs like muscle spasms and stiffness, and immunization history. Laboratory tests are generally not helpful for the diagnosis. ${ }^{2}$

Tetanus is a medical emergency and warrants immediate hospitalization and intensive care. The goals of the management are neutralization of circulating toxins with human tetanus immune globulin (TIG) or equine antitoxin (if human TIG is not available), stopping production of more toxins, with aggressive wound care and antibiotics, providing active immunization with a tetanus toxoid vaccine, and effective management of muscle spasms, respiratory failure, autonomic dysfunction, and other complications. ${ }^{5}$ Unfortunately, tetanus is most common in regions with lowest healthcare infrastructure. The mortality of NT in the developing countries in the absence of intensive care approaches nearly $100 \%$. Maternal tetanus carries an equally grave prognosis.

Given the dismal prognosis, there is a need to eliminate this dangerous disease by ensuring maternal tetanus immunization, safe delivery, and healthy umbilical cord care practices. Despite concerted efforts of the nations all over the world, global maternal and neonatal tetanus elimination (MNTE) remains elusive. With the setback in routine vaccination due to the recent COVID-19 pandemic, there is a need to revisit the strategies needed to attain the goal of MNTE.

\section{Definition of Neonatal and Maternal Tetanus}

Tetanus occurring during the neonatal age is termed NT. For surveillance purpose, a confirmed case of NT is defined by the World Health Organization (WHO) as

Any neonate who was normal and able to suck; and cry normally during the first two days of life AND later could not suck normally between 3 and 28 days of age AND who develops muscle stiffness and/or spasms.

Maternal tetanus is defined as tetanus occurring anytime during pregnancy or within 6 weeks of termination of pregnancy (either birth, miscarriage, or abortion). ${ }^{6}$

Neonatal tetanus elimination (NTE) is defined as fewer than 1 case of NT per 1000 live births in each district or equivalent administrative unit of a country in a year. There is no operational definition for maternal tetanus elimination (MTE) as such, and incidence of NT is used as a proxy indicator for MTE. ${ }^{7}$

\section{Magnitude of Neonatal and Maternal} Tetanus

The exact magnitude of neonatal and maternal tetanus is difficult to measure, as the disease is under-reported; the majority of cases occur in the most underprivileged areas, where home deliveries and unsafe delivery practices are common and the healthcare system is poorly developed. Reported cases, therefore, just represent the proverbial "tip of the iceberg". Mathematical models have been used to estimate the burden of MNT. $^{3}$ WHO estimated that 787,000 neonatal deaths occurred due to neonatal tetanus in 1988 and this reduced to 25,000 in 2018 (Figure 1). ${ }^{8-10}$ The actual data of burden of MT are not available as the reporting format does not exist in most countries. Despite tetanus being a notifiable disease across many nations, cases of MT as well as NT remain disguised.

\section{Need for Maternal and Neonatal Tetanus Elimination}

Even a single case of neonatal or maternal tetanus is significant, as achieving maternal and neonatal tetanus elimination (MNTE) is a proxy marker of a nation's robustness of three fundamental public health issues, viz the routine immunization program, quantum of available antenatal care, and clean and safe birth practices. The nations who are still to be validated for MNTE need to scrutinize their healthcare policies and consolidate their efforts in strengthening these issues, in a comprehensive manner.

\section{Maternal Neonatal Tetanus Elimination (MNTE) Strategy}

Clostridium tetani spores are difficult to kill and require autoclaving or prolonged exposure to sterilizing agents. The spores survive in soil, and their transmission does not require human contact. Because of the ubiquitous presence of tetanus spores in the soil and gut of many animal species, it is not possible to eradicate the source of infection. ${ }^{11}$

Neonatal tetanus was a significant public health issue in the late 1980s, and 787,000 neonatal deaths were attributed to it in $1988 .{ }^{6}$ Taking cognizance of the significant burden, the World Health Assembly in 1989 launched a global neonatal tetanus elimination (NTE) program to achieve elimination of NT in all countries by 1995 . Elimination of maternal tetanus was added to "NTE", and the program was rechristened in 1999 as "MNTE" by WHO, United Nations Children's Fund (UNICEF), 


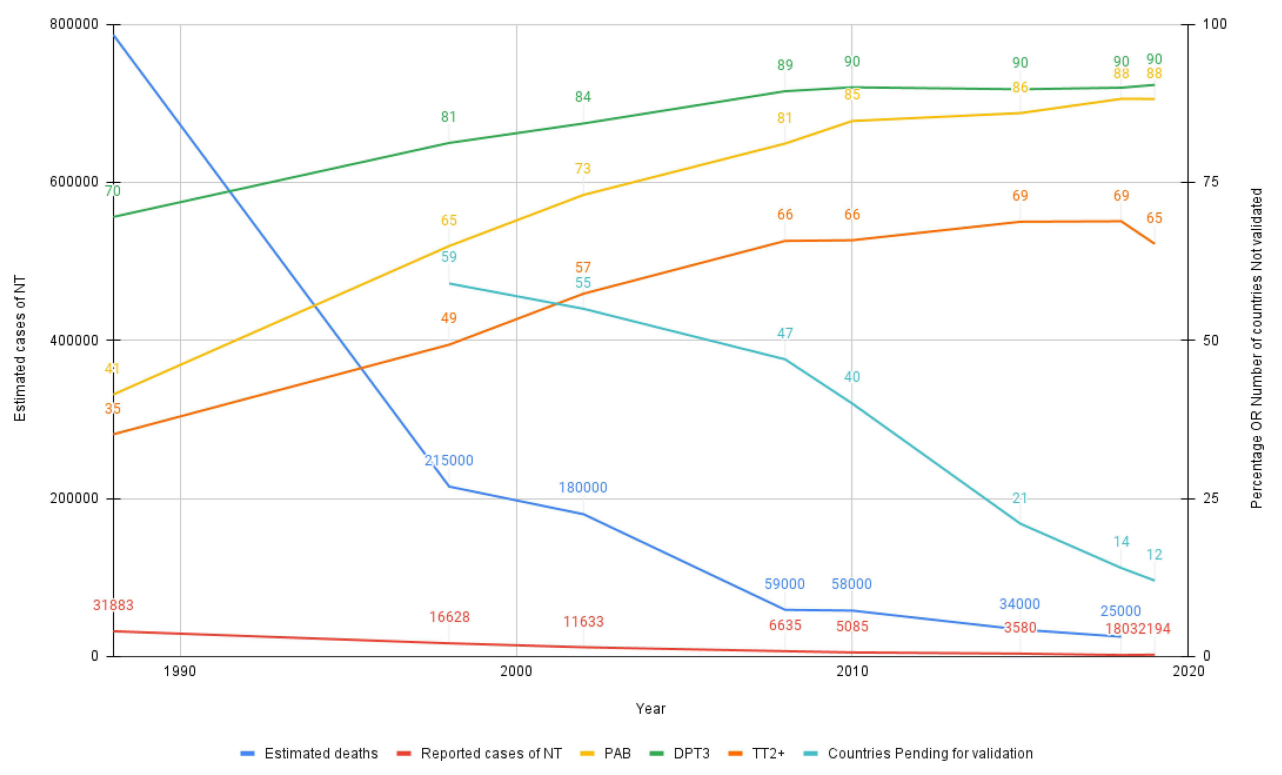

Figure I Global surveillance indicators for maternal and neonatal tetanus elimination. Data from these studies. ${ }^{8-10}$

and United Nation Fund for Population Activities (UNFPA). The goal of MNTE was to eliminate tetanus from the neonatal and maternal population in 59 priority countries initially by 2005 and later on extended to 2015 , but both deadlines were missed. ${ }^{12}$ A working group was constituted by the Strategic Advisory Group of Experts on Immunization (SAGE), WHO, in 2015 to scrutinize the reasons of missing of targets for MNTE. This group, on the basis of evidence, had set the year 2020 as the next target year of achieving MNTE in the remaining priority countries to align it with the Global Vaccine Action Plan (GVAP).$^{13}$ Unfortunately, MNT continues to be the invisible killer in 12 countries, viz Afghanistan, Angola, Central African Republic, Guinea, Mali, Nigeria, Pakistan, Papua New Guinea, Somalia, South Sudan, Sudan, and Yemen. ${ }^{8,14}$

\section{Strategies for Achieving MNTE}

There are three stages of MNTE: achieving, validating, and sustaining. After achieving elimination status, the country applies for the validation status. It is certified to have achieved validation for MNTE if the elimination status of NT $(<1$ case per 1000 live births) has been verified by district level data using core and supplementary indicators (Tables 1 and 2). It is of paramount importance to sustain MNTE status once it is achieved and validated. The implementation strategies differ in the countries, depending upon whether MNTE status is to be achieved or sustained.
The three-pronged elimination strategies which are used for achieving MNTE are rigorous immunization of women of reproductive age (15-49 years) with tetanus toxoid, strengthening of clean delivery services for pregnant women, and effective surveillance for the MNT. ${ }^{15}$

- Immunization of women of reproductive age (WRA) with TTCVs: Immunization against tetanus using TTCVs is the most important component for preventing MNT. Neonates and young infants born to mothers having antitetanus antibodies are automatically protected against tetanus by virtue of acquired maternal antibodies.

- Providing clean and safe birth practices by involving skilled birth attendants: Ensuring clean delivery practices during birth can prevent a significant number of cases of MNT, as the spores are naturally present in the soil. A clean and safe delivery is defined as a delivery conducted by a physician, nurse or midwife, or delivery at a healthcare facility. It could also be defined as per the country's national policy, where the institutional deliveries are lesser. The practicing of "cleans" during the birth also helps to reduce the incidence of MNT.

- Ensuring optimal surveillance for MNT: The presence of a sensitive surveillance system is the backbone for MNTE. Epidemiological analysis, setting up targets, execution of strategies, and evaluation of the success of the program all depend upon the quality of the NT surveillance. 
Table I District Level Core Indicators for Maternal and Neonatal Tetanus Surveillance

\begin{tabular}{|c|c|}
\hline Core Indicator & Remarks \\
\hline Reported NT cases & $\begin{array}{l}\text { Number of neonatal tetanus cases reported. } \\
\text { - If no case is present, "Zero reporting" is to be done. } \\
\text { - Exact number of cases are to be reported. } \\
\text { - In areas having high NT burden, sensitivity and reliability of the surveillance system should be ensured before } \\
\text { accepting. }\end{array}$ \\
\hline $\begin{array}{l}\text { Number of live births } \\
\text { (LBs) }\end{array}$ & To be derived from district level population estimates. \\
\hline $\begin{array}{l}\text { Reported NT rate/I000 } \\
\text { LBs }\end{array}$ & Incidence rate of neonatal tetanus cases reported. \\
\hline Quality of surveillance & $\begin{array}{l}\text { The reported data should be sensitive, which is defined as: } \\
\text { - Adequate number of reporting sites with representative distribution should be present, } \\
\text { - Presence of "zero" reporting, } \\
\text { - Completeness of reporting of at least } 80 \% \text { from all reporting sites, } \\
\text { - Conduction of review of records and/or of active NT surveillance, at least once annually. } \\
\text { - Functional community surveillance in rural areas. }\end{array}$ \\
\hline Clean delivery rate & $\begin{array}{l}\text { Defined as delivery assisted by skilled health personnel (physician, nurse, or midwife). } \\
\text { - Individual nationally accepted definition would also be acceptable. } \\
\text { - Definition should be clearly specified and used consistently for all districts. }\end{array}$ \\
\hline TT2+ coverage & $\begin{array}{l}\text { Proportion of pregnant women who received their second or higher TT dose during pregnancy in a given year. } \\
\text { Shortcoming of this indicator: } \\
\text { - The estimates are based on district administrative data, } \\
\text { - Under-estimating the true level of protection, } \\
\text { - Pregnant women having received } 5 \text { TT doses and did not get extra TT during pregnancy would be reported } \\
\text { missed, } \\
\text { - Re-starting of TT series and reporting it TTI, if TT immunization records unavailable, } \\
\text { - Supplemental doses not included where TT SIAs have been received, } \\
\text { - Booster doses received in childhood and adolescent age are not taken into account. }\end{array}$ \\
\hline
\end{tabular}

Notes: Data from the World Health Organization. ${ }^{6}$

Abbreviations: NT, neonatal tetanus; TT, tetanus toxoid; SIA, supplementary immunization activities.

\section{"High Risk" and "Low Risk" Area Approach}

It was seen that cases of MNT continued to be reported from areas which underperformed in routine immunization services and had a relatively less developed healthcare infrastructure. For this reason, the concept of targeting these "high-risk areas" was conceptualized by WHO. For achieving MNTE, the WHO operational algorithm classifies the districts or the administrative units into "High risk" and "Low risk" areas on the basis of the following 4 parameters: incidence of NT, the sensitivity of NT surveillance, the proportion of women who have received at least two doses of tetanus toxoid (TT2+), and the estimated clean delivery rate as shown in Figure 2. ${ }^{12}$ The countries are classified into class A, class B, and class
$\mathrm{C}$ on the basis of the number of high-risk districts, and number of beneficiaries who have received TT2+ (Table 3). ${ }^{10}$

- Low-risk areas: TTCVs are provided to the pregnant women during the routine antenatal visits, or at outreach centers or at fixed sites. Two doses of TTCV, one month apart, are administered in these areas and are sufficient to achieve the immunization for the current pregnancy; this interaction chance is also utilized to prime the pregnant women about the components of the clean delivery processes and healthy cord care practices.

- High-risk areas: These areas are chosen on the basis of thorough data review, in high-risk districts, and 
Table 2 District Level Supplemental Indicators for Maternal and Neonatal Tetanus Surveillance

\begin{tabular}{|c|c|}
\hline Supplemental Indicators & Remarks \\
\hline Protection at birth (PAB) & $\begin{array}{l}\text { Proportion of children protected against NT at the time of birth based on their mothers' complete TT } \\
\text { immunization history. } \\
\text { - Used by countries to complement TT2+. } \\
\text { - If PAB data are available, should be included in district review as well. }\end{array}$ \\
\hline SIA TT coverage (TTI, TT2, TT3) & $\begin{array}{l}\text { The proportion of women in the district having received } 2 \text { or } 3 \text { doses of TT during SIAs. } \\
\text { - Indication of the management of immunization services. }\end{array}$ \\
\hline DPTI coverage & Indicates access to immunization services and may indicate access to other health care. \\
\hline $\begin{array}{l}\text { Antenatal care coverage (I visit } \\
\text { minimum) }\end{array}$ & $\begin{array}{l}\text { The proportion of pregnant women having made at least one visit for antenatal care. } \\
\text { - Complements data on clean delivery. }\end{array}$ \\
\hline DPTI and DPT3 coverage & $\begin{array}{l}\text { Number of beneficiaries who have received three DPT of primary series. } \\
\text { - Indicates a well-managed immunization program and complements TT2+ coverage estimates. } \\
\text { - Indicates robust primary series vaccination program. }\end{array}$ \\
\hline DPT3-DPTI dropout rate & $\begin{array}{l}\text { Number of beneficiaries who did not receive DPT3 after receiving DPTI. } \\
\text { - Provides an indication of the management of immunization services. }\end{array}$ \\
\hline Urban vs rural status & Difficulty of terrain. \\
\hline \multicolumn{2}{|l|}{ Other Indicators } \\
\hline \multicolumn{2}{|c|}{$\begin{array}{l}\text { Measles vaccine and/or Bacillus Calmette-Guerin coverage, trained birth attendant coverage, women's literacy, \% population living in poverty an } \\
\text { other human development indicators, and health service access indicators such as population per health center and health centers } / \mathrm{km}^{2} \mathrm{may} \text { also b } \\
\text { used wherever available. }\end{array}$} \\
\hline
\end{tabular}

Notes: Data from the World Health Organization. ${ }^{6}$

Abbreviations: NT, neonatal tetanus; TT, tetanus toxoid; TT2+, proportion of pregnant women who received their second or higher TT dose during pregnancy in a given year; SIA, supplementary immunization activities; DPT, diphtheria, pertussis, tetanus.

identification of areas within the district where the access to routine vaccination is absent or very little, and that are characterized by unavailability of skilled birth attendants and antenatal care facility. In these areas, in addition to the routine immunization practices, supplementary immunization activities (SIAs) are carried out and TTCV immunization is provided to all women of reproductive age (WRA). At least three doses of TT/Td vaccines, irrespective of their previous vaccination status, are given at 0,1 month, and 6 months to at least $80 \%$ WRA. Local initiatives are also encouraged to enhance the practices for clean birth as well as hygienic cord care. Morocco was able to decrease the deaths due to NT by $90 \%$ from $20 \%$ to $2 \%$ of all neonatal deaths, using the high-risk area approach. ${ }^{15}$ Over a period of five years, Egypt was also able to cover all high-risk districts and achieve MNTE validation status by using the high-risk area approach. ${ }^{16}$

\section{Surveillance Indicators for Achieving MNTE}

During the process of MNTE, it is important to monitor the country's own performance. During the progress towards achieving the elimination, the total numbers of high-risk and low-risk districts in the country are estimated, and core as well as supplemental indicators are monitored in relation to the district level population estimates. The details of these indicators are provided in Tables 1 and 2.

\section{Quality of Surveillance Data}

Since the planning, execution, and assessment of MNTE depends upon the surveillance, the surveillance data should be "sensitive". ${ }^{6}$ WHO has recommended the following parameters to be used for labeling the surveillance as "sensitive": (1) presence of adequate number of reporting sites with representative distribution, (2) presence of "zero" reporting, (3) completeness of reporting of at least $80 \%$ from all reporting sites, (4) conduction of review of 

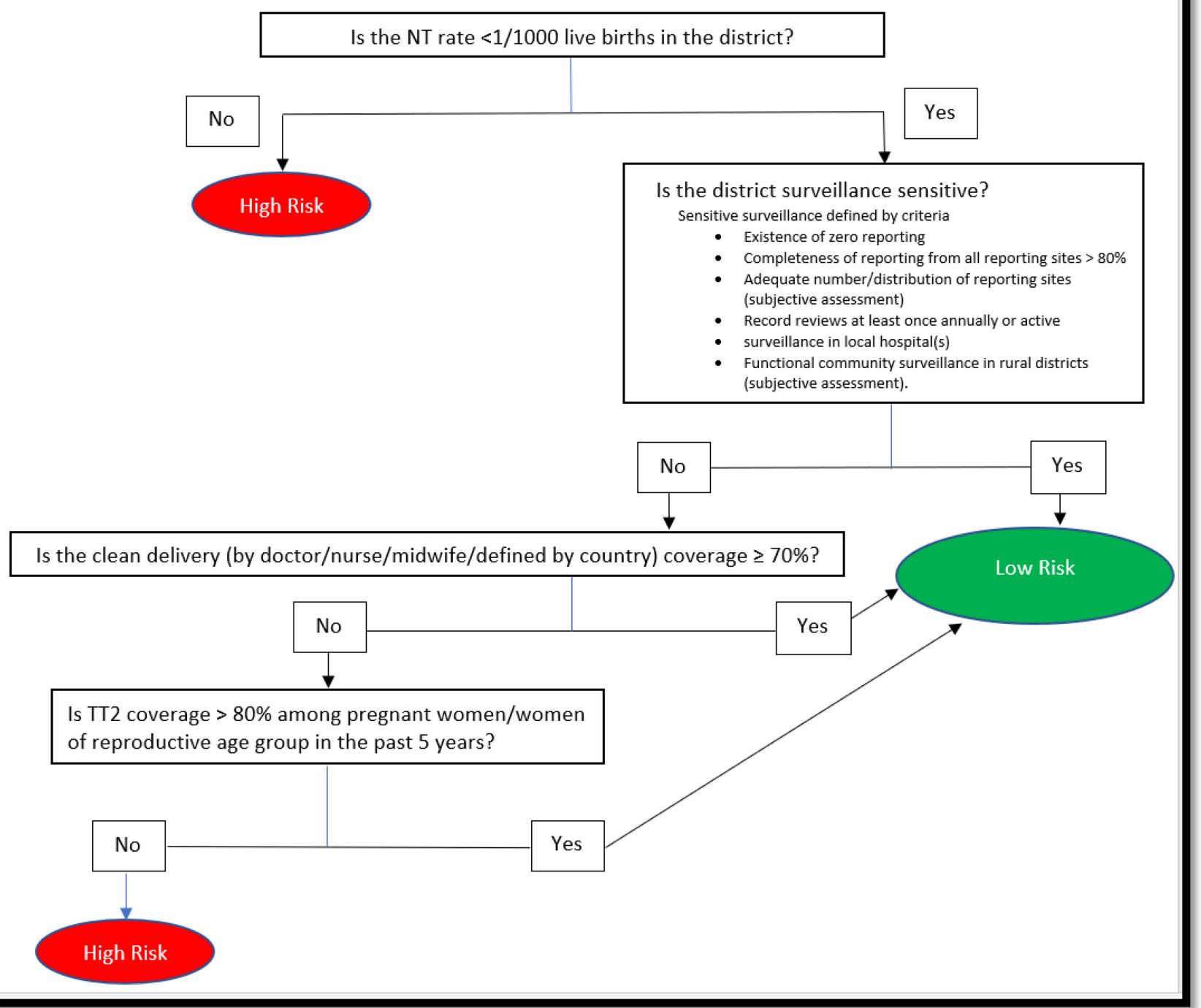

Figure 2 World Health Organization algorithm to identify high-risk districts for maternal and neonatal tetanus elimination surveillance. Notes: Data from the World Health Organization. ${ }^{66}$

records and/or of active NT surveillance, at least once annually, and (5) functional community surveillance in rural areas which have limited health facility access to ensure that neonatal deaths in rural areas are detected, reported, and investigated to rule out NT. Data from the lowest-performing districts are specially evaluated. ${ }^{6}$

Table 3 Classifications of Countries as per Maternal and Neonatal Tetanus Surveillance Indicators

\begin{tabular}{|l|l|l|l|}
\hline Parameter & Class A & Class B & Class C \\
\hline Number of High-Risk Districts & $<10 \%$ & $\mathrm{II}-50 \%$ & $>50 \%$ \\
\hline TT Coverage & $>70 \%$ & $50-70 \%$ & $<50 \%$ \\
\hline Estimated Time for Achieving MNTE & $\mathrm{I}$ year & 3 years & More than 3 years \\
\hline Countries as in 2020 & Afghanistan, Guinea, Pakistan & $\begin{array}{l}\text { Angola, Central African } \\
\text { Republic, Somalia, Sudan }\end{array}$ & $\begin{array}{l}\text { Mali, Nigeria, Papua New Guinea, } \\
\text { South Sudan, Yemen }\end{array}$ \\
\hline
\end{tabular}

Notes: Data from these studies. ${ }^{10,35}$

Abbreviation: MNTE, maternal and neonatal tetanus elimination. 
It is possible that the surveillance data are also underrepresented as the data belong to the districts which are low-performing and have comparatively less healthcare infrastructure. Moreover, a few of the indicators are subjective like "functional" community surveillance and "adequate number/distribution of reporting sites", and the interpretation can be different for different regions. Therefore, all other indicators as shown in Table 2 which are available at the time of analysis are also considered in addition to the surveillance data.

\section{Validation Process for MNTE}

Once a country believes that the elimination status has been achieved in all its districts, it applies to the WHO headquarters for MNTE validation. The validation process is carried out using uniform methods across the globe as specified by WHO. The complete district level raw data for several previous years are systematically reviewed by representatives of WHO, UNICEF, government agencies, and non-governmental organizations using the core and supplemental indicators to confirm the elimination. ${ }^{13}$ After completing the data review, the validation team reaches one of these three unanimous decisions and suggests actions: (a) MNT likely eliminated: Team deliberates if more information or surveys are needed, (b) MNT not eliminated: Corrective actions are recommended like execution of more SIAs, strengthening the outreach, (c) MNT likely eliminated: "Lot Quality Assurance" cluster sampling field surveys are then recommended in the districts which are at highest risk, to reach a consensus.

\section{Sustaining Phase}

The elimination of MNT is a dynamic process, and the countries which have achieved MNTE should continuously put in all efforts to sustain the status of MNTE. The ubiquitous nature of the spores may lead to resurgence of MNT whenever the guard is lowered by either decreased immunization coverage, or compromised safety and breach in the clean birth practices. ${ }^{16}$

\section{Likely Strategies to Sustain MNTE}

To sustain the elimination, modification and strengthening of the successful strategies used for achieving MNTE are essential. Sustaining MNTE requires a diligent and multifaceted approach. Improvement in the antenatal care; clean delivery with skilled personnel; protection at birth; and strengthening of routine immunization program for TTCV as shown in Figure 3 are the four essential pillars

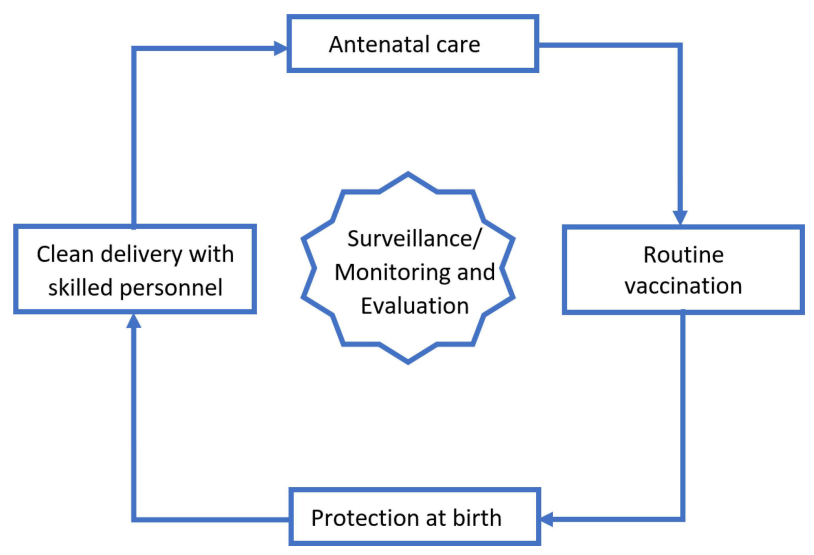

Figure 3 Strategies for sustaining maternal and neonatal tetanus elimination.

on which sustaining MNTE depends. WHO has proposed the following strategies for sustaining MNTE: ${ }^{12}$

1. Immunization with TTCV: Sustaining MNTE is possible only if instead of focusing on the WRA alone, long-term planning is also done to include all of the population for TTCV immunization. The already started practice of TTCV immunization to all pregnant women during the "achieving phase" (one of the strategies to achieve MNTE) would need strengthening. In addition, planning should be done to immunize future cohorts of WRA by administering 6 doses of TTCV ( 3 primary infant doses and 3 booster doses of TTCV) before adolescence. Most of the adult WRA population gets protection against tetanus if $90 \%$ of population has received 6 doses before adolescence. ${ }^{17}$

2. Antenatal screening of pregnant women for verification of TTCV: As compared to the "achieving phase" where the antenatal period was used to give TTCV, the strategy now should be to screen the pregnant women coming for ANC for confirmation of receiving of TTCV. The healthcare provider should ask the history of receiving of TTCV at the time of first ANC and document it. Eventually this should also render the SIA used for targeting highrisk cases unnecessary as most of the WRA would be already immunized against tetanus. This opportunity should also be used to sensitize the beneficiaries about safe birth and cord care practices.

3. Care at birth: Strategies should be put in place to provide increased access to skilled health personnel (SHP; earlier referred to as skilled birth attendant, SBA) at all births. All efforts should be made to provide clean birth 
(defined as delivery using hygienic practices and attended by competent maternal and newborn health personnel) in a healthcare facility or at home. Clean cord care practices should also be enhanced.

4. Increased surveillance: Periodic systematic review of the "sensitive" surveillance data is an integral component to sustain MNTE. It would help to timely identify the districts which are lowperforming, start early remedial actions wherever required, and evaluate the efficacy of the effectiveness of program.

\section{Role of TTCV Immunization}

WHO has recommended to replace standalone TT vaccine with Td vaccine in older children, adolescents, and WRA since 1988 to decrease the outbreaks of diphtheria, as the schedule of diphtheria vaccine is similar to that of tetanus. ${ }^{18}$ For MNTE to be achieved as well as sustained, at least $80 \%$ of pregnant women in every district should be completely protected against tetanus. All persons who have been immunized with 6 doses of TTCV, starting from infancy (or 5 doses if vaccinated after infancy), are considered fully protected against tetanus throughout their adulthood and beyond. The duration of protection after different doses is explained in Table 4.

All efforts should be made to vaccinate children with 3 primary infancy series TTCV starting at 6 weeks of age, with a minimum 4-week gap between any two doses, and subsequently three booster doses of the TTCV at 12-23 months, 4-7 years, and 9-15 years of age to all eligible children. There should be an optimal gap of 4 years between the booster doses. All countries should make efforts to reach the Global Vaccine Action Plan (GVAP) goal of $>90 \%$ coverage. ${ }^{19}$ Since the administration of six doses of TTCV to the beneficiaries provides potential lifelong immunity, different strategies have been tried to increase the vaccination coverage in infancy and at each booster dose. The "Reaching Every District (RED) approach" has been devised by WHO to increase immunization coverage in infants. ${ }^{20}$ Second year of life (2YL platform), day-care or school-based vaccination strategy, and integration of human papilloma virus (HPV) vaccine with TTCV are a few of the strategies which have specifically targeted first, second, and third booster, respectively. Piggybacking the HPV vaccine on TTCV is a strategy with dual benefits of decreasing cervical cancer risk as well maternal tetanus incidence, and seems promising. $^{21}$

\section{Strategy at Antenatal Care Contact}

With increasing the coverage of complete primary immunization, more pregnant women would now be fully protected against tetanus and hence would not require repeat TTCV. Therefore, screening pregnant women who have not received adequate TTCV has become more important. Additionally, the ANC visit should also be utilized to emphasize the importance of presence of SHP at the time of delivery, giving birth at a health facility, following clean cord care practices, and complete vaccination of infants. The vaccination status should be confirmed by vaccination card or history. If the pregnant woman has received 6

Table 4 Schedule of Tetanus Toxoid-Containing Vaccines in Unvaccinated Pregnant Women

\begin{tabular}{|l|l|l|}
\hline $\begin{array}{l}\text { Number of Total TTCV Doses } \\
\text { Received }\end{array}$ & Timing of Administration & $\begin{array}{l}\text { Expected Duration of Protection } \\
\text { Corresponding to PAB }\end{array}$ \\
\hline One & At first contact or as early in pregnancy as possible & None \\
\hline Two & $\begin{array}{l}\text { At least } 4 \text { weeks after TTCVI (at the latest 2 weeks } \\
\text { prior to birth) }\end{array}$ & I-3 years \\
\hline Three & $\begin{array}{l}\text { At least } 6 \text { months after TTCV2, or during subsequent } \\
\text { pregnancy }\end{array}$ & At least 5 years \\
\hline Four & $\begin{array}{l}\text { At least I year after TTCV3, or during subsequent } \\
\text { pregnancy }\end{array}$ & At least I0 years \\
\hline Five & $\begin{array}{l}\text { At least I year after TTCV4, or during subsequent } \\
\text { pregnancy }\end{array}$ & For all childbearing age and much of adulthood \\
\hline
\end{tabular}

Notes: Data from the World Health Organization. ${ }^{1212}$

Abbreviations: TTCV, tetanus toxoid-containing vaccines; PAB, proportion of children protected against NT at the time of birth. 
Table 5 Schedule of Tetanus Toxoid-Containing Vaccines in Partially Immunized Pregnant Women

\begin{tabular}{|c|c|c|c|c|c|c|c|}
\hline \multirow{2}{*}{$\begin{array}{l}\text { Age of Recipient at } \\
\text { Previous TTCV } \\
\text { Vaccination }\end{array}$} & \multicolumn{3}{|c|}{$\begin{array}{l}\text { Number of Total Doses } \\
\text { Received }\end{array}$} & \multicolumn{4}{|c|}{ Vaccination Schedule of Remaining TTCV } \\
\hline & Primary & Booster & Total & $\begin{array}{l}\text { At } \\
\text { Current } \\
\text { Contact }\end{array}$ & Later & Total & Timing of Subsequent Doses \\
\hline Infancy & 3 & 0 & 3 & 1 & 2 & 3 & $\begin{array}{l}\text { Minimum 4-week interval between first two doses (but } \\
\text { at least } 2 \text { weeks prior to delivery), next dose after } \\
\text { I year of last dose taken }\end{array}$ \\
\hline $\begin{array}{l}\text { Early childhood/ } \\
\text { school age }\end{array}$ & 3 & 1 & 4 & I & I & 2 & Next dose after I year of last dose taken \\
\hline School age & 3 & 2 & 5 & I & 0 & 1 & None required (fully protected) \\
\hline Adolescence & 3 & 3 & 6 & 0 & 0 & 0 & None required (fully protected) \\
\hline
\end{tabular}

Notes: Data from the World Health Organization. ${ }^{1 / 2}$

Abbreviation: TTCV, tetanus toxoid-containing vaccines.

doses (or 5 doses after infancy, as described above), she does not require further TTCV. However, it should be clearly documented in the card that she is already fully vaccinated with TTCV. If the vaccination card or history shows that the woman is partially/not vaccinated, TTCV should be administered as per schedule given in Tables 4 and 5. Care should be taken that the last dose of TTCV should be given at least 2 weeks prior to delivery.

The mother of a neonate who is diagnosed to be suffering from NT should also receive doses of TTCV as unvaccinated women: first dose at first contact, second dose after 4 weeks of first dose, and third dose after 6 months of second dose and subsequently as in Table 5. Other partially/not vaccinated women requiring TTCV from same geographical area should also be identified and vaccinated as described in Tables 4 and $5 .{ }^{12}$

\section{Clean Births}

Tetanus is acquired by the entry of the tetanus spores through contaminated wounds, therefore ensuring clean birth practices would be able to effectively decrease the incidence of MNT, even if the TTCV immunization coverage is less than optimum in certain areas. Clean birth practices include conducting of delivery using hygienic practices and providing clean cord care. These practices can only be ensured by presence of SHP at the time of every delivery. ${ }^{22}$ Every Newborn Action Plan (ENAP), the Global Strategy for Women's, Children's and Adolescent Health, the Sustainable Development Goals, and Ending Preventable Maternal Mortality Initiative all support the presence of the competent health professional at the time of delivery to decrease the neonatal mortality rate (NMR). ${ }^{23-26}$

Differently summarized "cleans" (Three cleans, Five cleans, and Six cleans) have been described in the literature, which emphasize the importance of clean birth practices. $^{7,27,28}$ Common components are clean hands, clean surface, clean cord cut, clean cord tie, and clean cord care. The details of these "cleans" are described in Table 6. Clean birth kits (CBK) are kits containing the disposables which are essential for conducting clean delivery. ${ }^{29}$ Different types of CBK are available for home deliveries (with as well as SHP) and primary health facilities and referral centers. They can be used by mothers as well as SHP. The United Nations Population Fund and WHO have standardized the contents of the CBK and also developed a safe childbirth checklist and an implementation guide to improve the quality of care provided to parturients in health facilities. $^{30,31}$

Many communities of the world traditionally apply various harmful substances like cow dung, herbs, oils, spices, etc. on the cord. Clean birth practices have been shown to decrease the incidence of neonatal tetanus by $55-99 \% .^{13}$ It also decreases the incidence of neonatal sepsis and overall neonatal mortality. WHO recommends dry cord care in neonates born in hospitals and communities having low NMR, and a chlorhexidine digluconate $7.1 \%$ (equivalent to $4 \%$ chlorhexidine) once-daily application on the umbilical cord during the first week of life in community settings where the NMR is more than $30 .^{32}$ 
Table 6 "Cleans" to Be Followed During the Perinatal Period

\begin{tabular}{|l|l|}
\hline Clean hands of birth & $\begin{array}{l}\text { Protects the mother and the neonate from infections and is a prerequisite for ensuring a safe delivery. } \\
\text { attendant }\end{array}$ \\
$\begin{array}{ll}\text { - Clean hands with soap and clean water. } \\
\text { - Hand hygiene is to be done before the delivery. } \\
\text { - Following five moments of hand hygiene are recommended: } \\
\text { (before touching the patient, before clean or aseptic procedure, after exposure to body fluid, after touching the } \\
\text { patient, after touching the patients' surroundings). }\end{array}$ \\
\hline Clean delivery surface & $\begin{array}{l}\text { Protects the neonate and mother from exposure to contaminated surface and infections. } \\
\text { - For the mother to lie upon. } \\
\text { - To maintain clean birth canal and perineum. }\end{array}$ \\
\hline Clean cord cut & $\begin{array}{l}\text { New razor blade or new sharp instrument. } \\
\text { - Original packing is opened. }\end{array}$ \\
\hline Clean cord ties & $\begin{array}{l}\text { Use clean or sterile thread or narrow tape. } \\
\text { - Tie the umbilicus tightly. } \\
\text { - Keeps the stump healthy. }\end{array}$ \\
\hline $\begin{array}{l}\text { Chlorhexidine cord care in community with high neonatal mortality settings. } \\
\text { - Dry cord care is recommended for those born in health facilities, and with low mortality settings. }\end{array}$
\end{tabular}

\section{Knowing That MNTE is Being Sustained}

Sustained MNTE can be monitored by conducting the standalone post-validation assessment, or by checking of the MNTE status as an integral part of the review process of any health program like the annual vaccine-preventable disease (VPD) data desk review, and national immunization program review. Additionally, special surveys need to be conducted to reconfirm validation status in selected districts. The objective is to maintain the MNTE status (less than 1 NT case/1000 live births/district) and to immediately launch corrective actions if any shortcoming is detected. ${ }^{15}$

In the annual VPDs data desk review, the key indicators of MNTE are actively sought from the full VPDs data. WHO has recommended that each country should conduct an annual data review of the VPDs as this will enable "no separate data collection for MNT". No additional funds need to be allocated for surveillance of MNTE once the system of collecting VPD data is established. It may even save the need of post-validation assessment if the data for neonates protected at birth (PAB) for tetanus and deliveries conducted at a health facility are available. But the district level data from low-performing districts may not be representative of the actual situation. ${ }^{12,15}$

In the assessment through the Expanded Program of Immunization Review, the variables required for monitoring MNTE could be included as an integral part of it. The combined data collected through field visits would then be analyzed to derive MNTE-specific indicators. The disadvantages are that these reviews are performed at intervals of three to five years, and highquality data from low-performing districts may not be available.

In the post-validation assessment for MNTE, the national surveillance data collected from all the districts are elaborately desk reviewed to confirm whether the MNTE indicators are sustained or not. The lowperforming districts which are at risk of resurgence of MNT are identified, and field surveys and interviews are planned to reconfirm the MNTE indicators. The root cause analysis of the issues hindering the implementation of MNTE is conducted, and corrective actions are initiated, wherever required. This method is most rigorous and directly linked to action, focuses only on MNTE, but is costly and requires meticulous planning to execute.

Estimation of tetanus antibodies by population-based cluster surveys can also give an objective measure of the immunity status of any population. Serosurveys for tetanus have been recommended by WHO SAGE in the countries where it is feasible to validate the risk status obtained through other data sources and accordingly plan the immunization strategy. ${ }^{6}$ Presence of seroprotection (defined as anti-tetanus antibody $\geq 0.1 \mathrm{IU} / \mathrm{mL}$ by ELISA) in more than 
Table 7 Timeline of Countries Achieving Maternal and Neonatal Tetanus Elimination Validation

\begin{tabular}{|l|l|}
\hline Year & Countries \\
\hline 2000 & Zimbabwe \\
2001 & Namibia \\
2002 & Malawi, South Africa \\
2003 & Eritrea \\
2004 & Rwanda \\
2005 & Nepal, Togo, Vietnam \\
2007 & Egypt, Zambia \\
2008 & Bangladesh \\
2009 & Burundi, Comoros, Congo, Turkey \\
2010 & Benin, Mozambique, Myanmar \\
2011 & Ghana, Liberia, Senegal, Uganda \\
2012 & Burkina Faso, Cameroon, China, G. Bissau, Tanzania, Timor Leste \\
2013 & Côte d'lvoire, Gabon, Iraq, Laos PDR, Sierra Leone \\
2014 & Madagascar \\
2015 & Cambodia, India, Mauritania \\
2016 & Eq. Guinea, Indonesia, Niger \\
2017 & Ethiopia, Haiti, Philippines \\
2018 & Kenya \\
2019 & Democratic Republic of Congo, Chad \\
\hline
\end{tabular}

Notes: Afghanistan, Angola, Central African Republic, Guinea, Mali, Nigeria, Pakistan, Papua New Guinea, Somalia, South Sudan, Sudan, and Yemen are the twelve countries who still are short of MNTE validation.

$80 \%$ of the population is shown to be compatible with MNTE. ${ }^{33}$ It could also be clubbed with other surveys like district health surveys, multiple indicator cluster survey, or vaccination coverage surveys. The irregular frequency, high cost, and logistics involved have resulted in this method being less commonly used.

\section{Current Status}

Despite missing the deadline of 2015 as elimination target, MNTE has been a huge success, and there has been a significant fall in the MNT cases and tetanus deaths. It is indeed a major accomplishment that 47 out of 59 highpriority countries were able to achieve validation of the MNTE status by the end of December 2019. DRC and Chad are the latest to achieve validation in $2019 .{ }^{8}$ The chronological year-wise validation details are given in Table 7., ${ }^{8,15}$ The overall TT2+ coverage has increased from $13.79 \%$ to $65.27 \%$, neonates having $\mathrm{PAB}$ has increased from average $12 \%$ to $88 \%$, which is a 6.5 -fold increase over 40 years, ${ }^{34,35}$ and global coverage of third-dose DPT has more than doubled in the last four decades. ${ }^{36}$ The region-wise data for these indicators are given in Figure $4 .{ }^{35}$ There has been a reduction of $88 \%$ in estimated deaths due to NT between
1988 and 2015, and 93\% reduction in global reported cases of NT from 1988 to 2019 as shown in Figure 1. Although not represented by any global estimates, the MT is also expected to follow the decline as per NT, as the improvement in both entities is governed by the same principles, viz. maternal vaccination and clean and safe deliveries.

Afghanistan, Angola, Central African Republic, Guinea, Mali, Nigeria, Pakistan, Papua New Guinea, Somalia, South Sudan, Sudan, and Yemen are the twelve countries who still have not received MNTE validation. It is promising to note that these countries are also trying, and specific regions of these countries like the Punjab province of Pakistan, southern regions of Mali, and the South East zone of Nigeria have been able to attain validation. ${ }^{8}$

\section{Barriers to Achieving Targets of MNTE}

Although specific reasons for not achieving MNTE may vary, they are usually from the constellation of sub-optimal healthcare infrastructure, less access to political and financial support from the governments and international funding agencies, inequities in immunization coverage, and below par NT surveillance. ${ }^{12,19,37}$ Regional or political conflicts, national security issues, and vaccine hesitancy in populations also decrease the universal immunization access. The lack of prioritization of MNT also often diverts the funds from MNTE strategies to other diseases. Poverty, illiteracy, and unawareness about the protective practices also lead to unsafe and non-clean deliveries and cord care practices. ${ }^{19,36,37}$

In Afghanistan, a conflict-ridden country with difficult terrain and gender bias, TTCV coverage was found to be insufficient in $46 \%$ of women; $38.1 \%$ of women did not receive any $\mathrm{ANC}^{38}$ The percentage of home deliveries in rural and urban Afghan women was $75 \%$ and $35 \%$, respectively. $^{39}$ An audit from Guinea and another from Pakistan pointed towards association of NT with low TTCV coverage, poor ANC, and unhygienic cord care practices. ${ }^{40,41}$ Reports from the Pakistan Demographic and Health Survey (2017-18) show that traditional birth attendants or dais attend only $24 \%$ of the deliveries, and only two-thirds of deliveries take place in health facilities. ${ }^{42}$ In South Sudan $91 \%$ of deliveries occur at home without access to a SBA and often in an unhygienic environment. ${ }^{43}$ The African countries which are yet to achieve MNTE validation status still have low delivery by SHP (range 19-62\%), lower PAB in 2015 (69\%), lower 

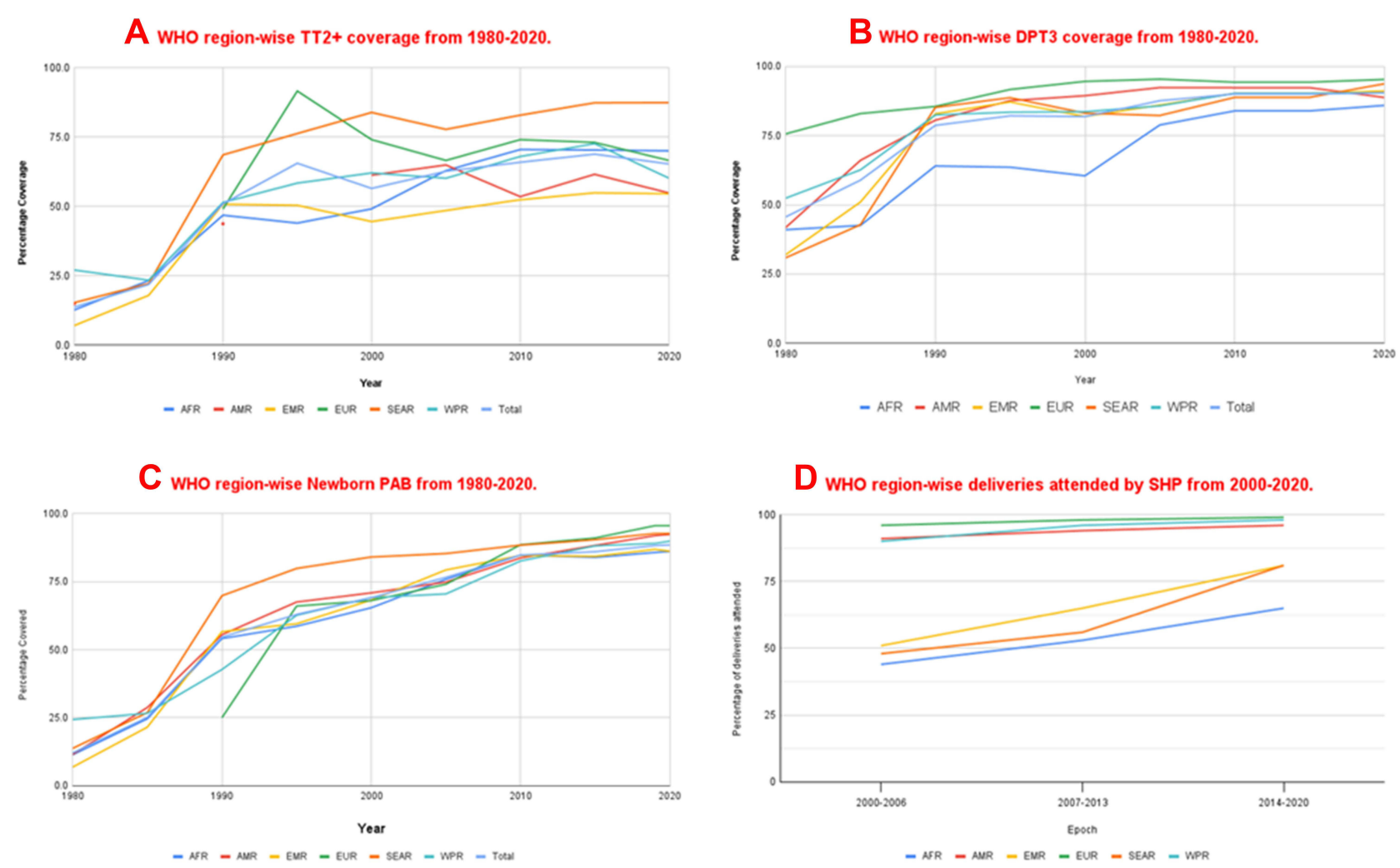

Figure 4 WHO region-wise surveillance indicators for MNTE. (A) WHO region-wise TT2+ coverage from 1980-2020. (B) WHO region-wise DPT3 coverage from 19802020. (C) WHO region-wise newborn PAB from 1980-2020. (D) WHO region-wise deliveries attended by SHP from 2000-2020.

Abbreviations: WHO, World Health Organization; MNTE, maternal and neonatal tetanus elimination; TT2+, proportion of pregnant women who received their second or higher TT dose during pregnancy in a given year; DPT3 coverage, number of beneficiaries who have received three DPT of primary series; PAB, protected at birth; AFR, The African Region; AMR, The Region of the Americas; EMR, The Eastern Mediterranean Region; EUR, The European Region; SEAR, The South-East Asia Region; WPR, The Western Pacific Region.

TT2+ coverage (64\%), lower DPT3 coverage $(<90 \%)$, and do not have provision of the recommended three TTCV boosters in the immunization schedule. ${ }^{44}$ Security concerns, inaccessibility, and competing priorities also play a role in Pakistan, South Sudan, Somalia, Nigeria, and Yemen. ${ }^{45}$ Funding is another important limiting factor, as huge financial support is needed to achieve MNTE in the countries who require maximum uplift of manpower and healthcare infrastructure.

In last two years, the COVID-19 pandemic has also resulted in decreased vaccination, more home deliveries, less SIAs, and diversion of resources, thereby causing a setback to the MNTE strategies. ${ }^{46}$ Even developed countries like USA witnessed a more than $50 \%$ decline in childhood vaccinations during the COVID-19 pandemic. ${ }^{47}$ Singapore, a country with a very robust healthcare system, also witnessed up to $10.3 \%$ drop in childhood vaccinations for 'diphtheria-tetanus-pertussis-inactivated polio-Haemophilus influenza vaccine. ${ }^{48}$ It is likely that low-resource countries where vaccination programs fared even worse are likely to see a surge in vaccine-preventable diseases in the times to come.

\section{Strategies for Achieving MNTE in Future}

Maternal and neonatal tetanus continues to be a ghost killer in not only these 12 countries but also elsewhere as most of the MNT cases may still go unreported. It is therefore pertinent to strengthen the pillars for achieving MNTE, viz TTCV coverage, clean birth practices, and improved surveillance. Achieving these in the countries which have still to be MNTE-validated would require more than the usual measures as most of these are having some major issues like insecurity, war etc. A MNT Plan of Action needs to be formulated by each country to combat MNT using regionally effective strategies. Flexible, multifaceted, and innovative approaches would have to be adopted to reach the goal. 
African countries like Eritrea and Liberia were able to decrease the incidence of MNT with training of traditional birth attendants to follow safe perinatal practices alone. ${ }^{49,50}$ Innovative ideas like administration of TTCV by a heat-stable prefilled injection device (UniJect) have been tried in Mali and Mozambique who had fewer healthcare workers. ${ }^{51,52}$ These syringes could be used by community volunteers without much training. Various global agencies like UNICEF, UNFPA, and The Kiwanis International have recently collaborated to launch "The Eliminate Project" for achieving MNTE. Some regions of Pakistan, Nigeria, and Mali have achieved validation through extensive SIAs using this project. ${ }^{53}$ More such activities would be required to cover all the high-risk areas of these countries. Since the SIAs lead to protection for five years, follow-up doses may need to be given in a phased manner. It definitely requires strong political will, logistic support, financial support, and microplanning for carrying out strategies for MNTE.

While the COVID-19 pandemic has hit the routine vaccination ${ }^{54}$ as well as several surveillance programs, ${ }^{55}$ it has another side to it as well. People have realized the need for vaccination and the benefits of vaccination in disease prevention. It is likely that in times to come, vaccine hesitancy will decrease and countries can capture the opportunity to revitalize post-COVID vaccination programs.

\section{Where We Stand}

Although the MNTE program has been a success in 47 out of the 59 priority countries listed by WHO, sustaining the elimination of MNT is challenging. Although the remaining 12 countries have shown improving trends as shown by the surveillance indicators, there is still a long way to go to achieve global elimination. This will require identifying the most vulnerable populations, microplanning at all levels of health care, involvement of local community support, tackling the implementation gap, strong political will, good financial support, and continued robust surveillance.

\section{Disclosure}

The authors report no conflicts of interest in this work.

\section{References}

1. Farrar JJ, Yen LM, Cook T, et al. Tetanus. J Neurol Neurosurg Psychiatry. 2000;69:292-301. doi:10.1136/jnnp.69.3.292

2. Link E, Edelmann L, Chou JH, et al. Tetanus toxin action: inhibition of neurotransmitter release linked to synaptobrevin proteolysis. Biochem Biophys Res Commun. 1992;189(2):1017-1023. doi:10.1016/0006291X(92)92305-H
3. Roper MH, Vandelaer JH, Gasse FL. Maternal and neonatal tetanus. Lancet. 2007;370(9603):1947-1959. doi:10.1016/S0140-6736(07) 61261-6

4. Patel JC, Mehta BC. Tetanus: study of 8697 cases. Indian J Med Sci. 1999;53:393-401.

5. Bassin SL. Tetanus. Curr Treat Options Neurol. 2004;6:25-34. doi:10.1007/s11940-004-0036-1

6. World Health Organization. Neonatal tetanus: vaccine preventable diseases surveillance standards [Internet]. Available from: https:// www.who.int/publications/m/item/vaccine-preventable-diseasessurveillance-standards-neonatal-tetanus. Accessed July 11, 2021.

7. Neonatal tetanus elimination field guide, 2nd edition PAHO/WHO | Pan American Health Organization [Internet]. Available from: https:// www.paho.org/en/documents/neonatal-tetanus-elimination-fieldguide-2nd-edition. Accessed July 11, 2021

8. Njuguna HN, Yusuf N, Raza AA, et al. Progress toward maternal and neonatal tetanus elimination - worldwide, 2000-2018. MMWR Morb Mortal Wkly Rep. 2020;69:515-520. doi:10.15585/ mmwr.mm6917a2

9. Tetanus [Internet]. Available from: https:/www.who.int/westernpaci fic/health-topics/tetanus. Accessed July 16, 2021.

10. World Health Organization, United Nations Population Fund \& United Nations Children's Fund (UNICEF). Maternal and neonatal tetanus elimination by 2005: strategies for achieving and maintaining elimination. World Health Organization; 2002. Available from: https://apps.who.int/ iris/handle/10665/69618. Accessed July 16, 2021.

11. Thwaites CL, Loan HT. Eradication of tetanus. $\mathrm{Br}$ Med Bull. 2015;116:69-77.

12. World Health Organization. Protecting all against tetanus: guide to sustaining maternal and neonatal tetanus elimination (MNTE) and broadening tetanus protection for all populations. World Health Organization; 2019. Available from: https://apps.who.int/iris/handle/ 10665/329882. Accessed July 16, 2021.

13. World Health Organization. Report of the SAGE Working Group on Maternal and Neonatal Tetanus Elimination and Broader Tetanus Prevention; September, 2016.

14. World Health Organization. Global vaccine action plan monitoring, evaluation \& accountability: secretariat annual report 2020. Available from: https://www.who.int/publications/i/item/global-vaccine-actionplan-monitoring-evaluation-accountability-secretariat-annual-report -2020. Accessed October 3, 2021.

15. World Health Organization. Validation of maternal and neonatal tetanus elimination including a guide to the use of lot quality assurance - cluster sample surveys to assess neonatal tetanus mortality [Internet]. TechNet-21. Available from: https://www.technet-21.org/ en/library/main/6589-validation-of-maternal-and-neonatal-tetanuselimination-including-a-guide-to-the-use-of-lot-quality-assurance- $\%$ E2\%80\%93-cluster-sample-surveys-to-assess-neonatal-tetanusmortality. Accessed July 11, 2021.

16. World Health Organization. Validation of neonatal tetanus elimination in Egypt by lot quality-assurance cluster sampling. Wkly Epidemiol Rec. 2007;82:237-242.

17. World Health Organization. Tetanus vaccines: WHO position paper, February 2017 - recommendations. Vaccine. 2018;36:3573-3575. doi:10.1016/j.vaccine.2017.02.034

18. World Health Organization. Diphtheria vaccine: WHO position paper, August 2017 - recommendations. Vaccine. 2018;36 (2):199-201. doi:10.1016/j.vaccine.2017.08.024

19. World Health Organization. Global Vaccine Action Plan [Internet]. Available from: https:/www.who.int/teams/immunization-vaccines-andbiologicals/strategies/global-vaccine-action-plan. Accessed July 21, 2021.

20. Vandelaer J, Bilous J, Nshimirimana D. Reaching Every District (RED) approach: a way to improve immunization performance. Bull World Health Organ. 2008;86:A-B. doi:10.2471/ BLT.07.042127 
21. Dunne EF, Markowitz LE, Saraiya M, et al. Centers for Disease Control and Prevention (CDC). CDC grand rounds: reducing the burden of HPV-associated cancer and disease. MMWR Morb Mortal Wkly Rep. 2014;63(4):69-72.

22. World Health Organization. Definition of skilled health personnel providing care during childbirth [Internet]. Available from: http:// www.who.int/reproductivehealth/publications/statement-competentmnh-professionals/en/. Accessed July 21, 2021.

23. World Health Organization. Every newborn action plan [Internet]. Available from: https://www.who.int/initiatives/every-newbornaction-plan. Accessed July 16, 2021.

24. World Health Organization. Global strategy for women's, children's and adolescents' health data portal [Internet]. Available from: https:// www.who.int/data/maternal-newborn-child-adolescent-ageing/docu ments/mca. Accessed July 16, 2021.

25. World Health Organization. Health in 2015: from MDGs, millennium development goals to SDGs, sustainable development goals [Internet]. World Health Organization; 2015 [cited July 16, 2021]. Available from: https://apps.who.int/iris/handle/10665/200009. Accessed July 16, 2021.

26. World Health Organization. Strategies towards ending preventable maternal mortality (EPMM). World Health Organization; 2015 Available from: https://www.who.int/reproductivehealth/topics/mater nal_perinatal/epmm/en/. Accessed August 8, 2021.

27. Care of a normal newborn baby. NNF teaching aids: newborn care. Available from: http://www.newbornwhocc.org/pdf/teaching-aids/nor malnewborn.pdf. Accessed August 8, 2021.

28. World Health Organization. Opportunities for Africa's newborns: practical data, policy and programmatic support for newborn care in Africa [Internet]. Available from: https://www.who.int/pmnch/media/ publications/africanewborns/en/. Accessed July 11, 2021.

29. Blencowe H, Cousens S, Mullany LC, et al. Clean birth and postnatal care practices to reduce neonatal deaths from sepsis and tetanus: a systematic review and Delphi estimation of mortality effect. BMC Public Health. 2011;11(Suppl 3):S11. doi:10.1186/1471-2458-11-S3-S11

30. United Nations Population Fund. Reproductive Health Kits Management Guidelines for Field Offices [Internet]. Available from: https://www.unfpa.org/resources/reproductive-health-kitsmanagement-guidelines-field-offices. Accessed July 21, 2021.

31. World Health Organization. Before birth: WHO safe childbirth checklist. World Health Organization; 2015. Available from: https:// apps.who.int/iris/handle/10665/199179. Accessed August 8, 2021.

32. World Health Organization. WHO recommendations on newborn health: guidelines approved by the WHO guidelines review committee [Internet]. Available from: https://www.who.int/publicationsdetail-redirect/WHO-MCA-17.07. Accessed July 21, 2021.

33. World Health Organization. Recommendations to assure the quality, safety, and efficacy of DT-based combined vaccines. Table 6.2 in: WHO expert committee on biological standardization, sixty-third report. Annex 6 (WHO Technical Report Series, No.980. Geneva: World Health Organization; 2014. Available from: http://www.who.int/biologicals/WHO_TRS_980_WEB.pdf. Accessed July 10, 2021.

34. World Health Organization. Neonates protected at birth against neonatal tetanus (PAB) (\%) [Internet]. Available from: https://www.who. int/data/gho/data/indicators/indicator-details/GHO/neonates-protected -at-birth-against-neonatal-tetanus-(pab)-(-). Accessed July 21, 2021.

35. World Health Organization: Immunization, Vaccines And Biologicals. Vaccine preventable diseases vaccines monitoring system 2020 global summary reference time series: TT2+ [Internet]. Available from: https://apps.who.int/immunization_monitoring/glo balsummary/timeseries/tscoveragett2plus.html. Accessed July 16, 2021.
36. Galles NC, Liu PY, Updike RL, et al. Measuring routine childhood vaccination coverage in 204 countries and territories, 1980-2019: a systematic analysis for the Global Burden of Disease Study 2020, release 1. Lancet. 2021;398(10299):503-521. doi:10.1016/S0140-6736(21) 00984-3

37. World Health Organization. Meeting of the strategic advisory group of experts on immunization, October 2016 - conclusions and recommendations. Wkly Epidemiol Rec. 2016;91:561-582.

38. Sherley J, Newton S. The association between area of residence and sufficient antenatal tetanus vaccination in women ages 15-49 in Afghanistan: an analysis of the 2015 DHS dataset. Glob Health Res Policy. 2020;5:51. doi:10.1186/s41256-020-00180-1

39. Kim C, Saeed KMA, Salehi AS, et al. An equity analysis of utilization of health services in Afghanistan using a national household survey. BMC Public Health. 2016;16:1226. doi:10.1186/s12889-016-3894-z

40. Condé I, Cherif MS, Dahal P, et al. Neonatal and postneonatal tetanus at a referral hospital in Kamsar, Guinea: a retrospective audit of paediatric records (2014-2018). Int Health. 2021. doi:10.1093/ inthealth/ihab021

41. Iqbal S, Ali I, Ekmekcioglu C, Kundi M. Increasing frequency of antenatal care visits may improve tetanus toxoid vaccination coverage in pregnant women in Pakistan. Hum Vaccin Immunother. 2020;16:1529-1532. doi:10.1080/21645515.2019.1705693

42. National Institute of Population Studies - NIPS/Pakistan and ICF. Pakistan demographic and health survey 2017-18. Islamabad, Pakistan: NIPS/Pakistan and ICF; 2019. Available from: http://dhspro gram.com/pubs/pdf/FR354/FR354.pdf. Accessed August 8, 2021.

43. Maternal and Neonatal Tetanus is South Sudan's 'silent killer'. Aid \& International Development Forum (AIDF) [Internet]. Available from: http://www.aidforum.org/topics/health-and-wash/maternal-andneonatal-tetanus-is-south-sudans-silent-killer/. Accessed July 17, 2021.

44. Ridpath AD, Scobie HM, Shibeshi ME, et al. Progress towards achieving and maintaining maternal and neonatal tetanus elimination in the African region. Pan Afr Med J. 2017;27(Suppl 3):24. doi:10.11604/pamj.supp.2017.27.3.11783

45. UNICEF. Kiwanis international and the Kiwanis children's fund 2018 report. maternal and neonatal tetanus elimination initiative. Available from https://www.google.com/url?sa=t\&rct=j\&q= $\&$ e s r c $=\mathrm{s} \& \mathrm{~s}$ o u r c e $=$ w e b \& $\mathrm{c} \mathrm{d}=\& \mathrm{v}$ e $\mathrm{d}=$ 2ahUKEwj9ouDC5KHyAhVUT30KHTfwBaIQFnoECAYQAw\&u $\mathrm{rl}=$ https $\% 3 \mathrm{~A} \% 2 \mathrm{~F} \% 2 \mathrm{Fwww}$.eliogarozzo.it $\% 2 \mathrm{Fwp}-\mathrm{content} \%$ 2Fuploads\%2F2020\%2F03\%2F2018-Global-MNTE-AnnualReport-for-KI.pdf\&usg=AOvVaw0IVvXTmRxVezNKU6rJAVqX. Accessed August 8, 2021.

46. Causey K, Fullman N, Sorensen RJD, et al. Estimating global and regional disruptions to routine childhood vaccine coverage during the COVID-19 pandemic in 2020: a modelling study. Lancet. 2021;398:522-534. doi:10.1016/S0140-6736(21)01337-4

47. Brooks HE, McLendon LA, Daniel CL. The impact of COVID-19 on pediatric vaccination rates in Alabama. Prev Med Rep. 2021;22:101320. doi:10.1016/j.pmedr.2021.101320

48. Zhong Y, Clapham HE, Aishworiya R, et al. Childhood vaccinations: hidden impact of COVID-19 on children in Singapore. Vaccine. 2021;39:780-785. doi:10.1016/j.vaccine.2020.12.054

49. World Health Organization. Assessment of neonatal tetanus elimination in Eritrea. Wkly Epidemiol Rec. 2004;79:222-227.

50. World Health Organization. Validation of maternal and neonatal tetanus elimination in Liberia, 2011. Wkly Epidemiol Rec. 2012;87:169-176.

51. La D, Sanogo B, Millogo J, Diarra S, Plowman B Impact of delivery technologies on increased access. TT-Uniject report Mali. 2004.

52. World Health Organization. Validation of elimination: maternal and neonatal tetanus in Mozambique, 2010. Wkly Epidemiol Rec. 2011;86:481-488. 
53. Eliminating maternal and neonatal tetanus [Internet]. Kiwanis Available from: https://www.kiwanis.org/childrens-fund/eliminatingmaternal-and-neonatal-tetanus. Accessed July 22, 2021.

54. Ali I. Impact of COVID-19 on vaccination programs: adverse or positive? Hum Vaccin Immunother. 2020;16(11):2594-2600. doi: $10.1080 / 21645515.2020 .1787065$
55. Manyanga D, Masvikeni B, Kuloba M, Byabamazima C, Daniel F. Early effects of the COVID-19 pandemic on the acute flaccid paralysis surveillance in East and Southern African countries. Pan Afr Med J. 2021;39:147. doi:10.11604/pamj.2021.39.147.28884

\section{Publish your work in this journal}

Research and Reports in Tropical Medicine is an international, peerreviewed, open access journal publishing original research, case reports, editorials, reviews and commentaries on all areas of tropical medicine, including: Diseases and medicine in tropical regions; Entomology; Epidemiology; Health economics issues; Infectious disease; Laboratory science and new technology in tropical medicine;

Submit your manuscript here: http://www.dovepress.com/research-and-reports-in-tropical-medicine-journal
Parasitology; Public health medicine/health care policy in tropical regions; and Microbiology. The manuscript management system is completely online and includes a very quick and fair peer-review system. Visit http://www.dovepress.com/testimonials.php to read real quotes from published authors. 